\title{
REDES DE INOVAÇÃO EM CLUSTERS: O LOCAL E O GLOBAL NA CRIAÇÃO DE VANTAGEM COMPETITIVA
}

\author{
Aline Lourenço de Oliveira ${ }^{1}$ \\ Ari de Souza Soares² \\ Cleber Carvalho de Castro3 \\ Daniel Leite Mesquita ${ }^{4}$
}

\begin{abstract}
RESUMO
Clusters são formatos organizacionais que apresentam um entrelaçamento de relações entre agentes públicos e privados em torno de um segmento produtivo em uma determinada região, o que tem sido considerado como fonte de vantagem competitiva para empresas e regiões. Destaca-se neste modelo um ambiente favorável ao desenvolvimento da inovação e melhoria da qualidade e produtividade, especialmente por propiciar o surgimento de redes de inovação. O processo inovativo que lhes é favorável pode ser potencializado pelo envolvimento de universidade - empresa governo, componentes do Modelo Tríplice Hélice. O objetivo deste trabalho é apresentar os clusters inovativos como formatos capazes de proporcionar vantagem competitiva por meio da relação entre o local e o global. Considera-se que a extensão da rede e suas características afetam o desempenho do cluster na medida em que os ambientes se tornam mais incertos. Argumenta-se que a capacidade das empresas para gerenciar o equilíbrio entre a força e a articulação de uma rede aberta é uma fonte fundamental de desempenho sustentável e competitivo do cluster. Observa-se que as instituições públicas podem desempenhar um papel importante para facilitar o desenvolvimento de conhecimento novo e consequentemente da inovação. Este artigo teórico aborda as características dos clusters que influenciam a vantagem competitiva dos agentes que os constituem; apresenta os conceitos e características das redes de inovação e o papel da Tríplice Hélice na sua constituição; e, trata, também, do potencial do âmbito local em conferir vantagem competitiva em um contexto de globalização.
\end{abstract}

Palavras-chave: Cluster. Modelo Tríplice Hélice. Inovação.

\footnotetext{
${ }^{1}$ Mestre; e-mail: aoliveirah@gmail.com

${ }^{2}$ Mestre; e-mail: arisoares@gmail.com

${ }^{3}$ Doutor; e-mail: clebercastro@ufla.br

${ }^{4}$ Mestre; e-mail: mdleite@gmail.com
} 


\section{INTRODUÇÃO}

Os Clusters representam um dos arranjos organizacionais que foram destacados em meio às transformações sociais e de produção que marcaram o século XXI. Em sua estrutura, formada do entrelaçamento de relações entre agentes econômicos, políticos e sociais em torno de um segmento produtivo, emerge um alto potencial de formação de redes de inovação. Esta organização produtiva é responsável pelo desempenho e fortalecimento do cluster, uma vez que o alinhamento de interesses dos agentes vão além dos ganhos por escala e sinergia.

Este trabalho sustenta-se na necessidade de compreender o fenômeno das Redes de Inovação nas partes que o compõe, evitando a idéia de homogeneidade e abrindo espaço para diferenciar os papéis de cada agente nos arranjos inovativos. Assim, a questão a ser respondida é: como se estabelece a vantagem competitiva em clusters inovativos? O objetivo deste trabalho é apresentar os clusters inovativos como arranjos capazes de proporcionar vantagem competitiva por meio da relação entre o contexto local e o global.

Este artigo teórico está estruturado em cinco partes, incluindo esta introdução e as considerações finais. A segunda seção trata da abordagem de clusters e as características que influenciam a vantagem competitiva dos agentes que o constituem. Na sequência, exploram-se os conceitos e características das redes de inovação e o papel da tríplice hélice na sua constituição. Adiante, relaciona-se o potencial do âmbito local em conferir vantagem competitiva aos clusters em um contexto de globalização.

\section{VANTAGEM COMPETITIVA E CLUSTERS}

O aspecto da geografia das empresas é uma temática que tem sido explorada por diversas abordagens que ressaltam a importância da articulação das firmas enfocando a dimensão territorial, como por exemplo: cluster, distrito industrial, arranjo produtivo local, pólos e parques tecnológicos. A questão da geografia das empresas foi inicialmente tratada na economia ao longo dos primeiros cinquenta anos do século 20 , com destaque para o trabalho de Marshall (1920), sendo depois negligenciada e retomada na década de 90, principalmente em decorrência dos trabalhos de Porter (1999) sobre a competitividade regional.

Enfocar a questão da geografia das empresas como um fator relevante da vantagem competitiva, em uma época onde espaço e tempo se tornaram relativos, suscita um paradoxo. Contudo para Eisingerich, Bell e Tracey (2010), o debate acerca das aglomerações desperta atualmente o interesse acadêmico e político, no sentido de se identificar os fatores que sustentam o desempenho dessas estruturas dentro de três vertentes: sua eficiência econômica, sua dinâmica de transferência de conhecimento 
e o tipo de estrutura social à que essas aglomerações estão submetidas. Dessa forma, a similaridade de oportunidades e as características regionais podem se tornar um diferencial competitivo. Em outras palavras, de modo geral, todas as empresas podem se beneficiar da dinamicidade do ambiente de negócios, mas, destacar-se nele exige muito mais esforço, e neste caso, tomar como referência as competências da região onde se está instalado pode ser um forte diferencial.

Assim, os saberes locais passam a ser entendidos como uma fonte de vantagem competitiva. Entendendo que vantagem competitiva relaciona-se a capacidade da empresa de manter e desenvolver suas ações, o importante no "mercado não é de fato, a qualidade dos produtos e serviços e a atratividade dos preços, mas sim, a percepção de superioridade desses atributos em comparação aos do concorrente" (CHRISTENSEN, 1999, p. 109). Logo, a vantagem competitiva é importante porque é o que determina que uma empresa, através de seus produtos e serviços, seja escolhida em detrimento de outras.

Nesse sentido a abordagem dos aglomerados (Clusters) recebe um destaque especial. Ela dá proeminência aos benefícios, particulares e coletivos, que podem advir da articulação de agentes localizados territorialmente próximos. Conforme Albagli e Brito (2003, p. 7), "a aglomeração de empresas vem efetivamente fortalecendo suas chances de sobrevivência e crescimento, constituindo-se em importante fonte geradora de vantagens competitivas". Jenkins e Tallman (2010) apontam que, nessa abordagem, os Clusters podem promover uma "simbiose" para o desenvolvimento de novas capacidades e conhecimentos, porque eles são dinamizados tanto em seu interior quanto difundidos para empresas externas, promovendo assim, maior vantagem competitiva.

Dentro desta perspectiva, tanto o conceito anglo-americano de Cluster quanto a vertente nacional de Arranjo Produtivo Local ( $A P L)$ ou aglomerado, trazem a mesma ideia de interrelacionamento de agentes que acabam por constituir uma rede de troca e complementação em torno de uma atividade produtiva. O que pode ser verificado na definição dada por Porter (1999, p. 211), de que "aglomerado é um agrupamento geograficamente concentrado de empresas inter-relacionadas e instituições correlatas numa determinada área, vinculadas por elementos comuns e complementares".

De acordo com Albagli e Brito (2003, p.4), "a sua formação encontra-se, geralmente, associada a trajetórias históricas de construção de identidades e de formação de vínculos territoriais (regionais e locais), a partir de uma base social, cultural, política e econômica comum". Todavia, Amato Neto (2000) chama a atenção que clusters são formados apenas quando os aspectos: setorial e geográfico estão combinados, de forma que se não houver essa conjugação serão apenas empresas de produção em setores e geografia dispersas.

Segundo Albagli e Brito (2003), este tipo de formato organizacional pode ser caracterizado pela dimensão territorial; diversidade de atividades e atores econômicos, políticos e sociais; conhecimento tácito; inovação e aprendizado interativos; e, governança. 
Os aglomerados estão presentes em economias grandes e pequenas, em áreas rurais e urbanas e em vários níveis geográficos (países, estados, regiões metropolitanas e cidades) (DUTRA, 2010). A dimensão territorial em que está instalado constitui o recorte específico de análise e de ação política, definindo o espaço onde os processos produtivos, inovativos e cooperativos ocorrem. Porém, suas fronteiras raramente coincidem com os sistemas padronizados de classificação setorial, podendo se estender ao longo de cidades ou até mesmo países.

Os clusters podem envolver diversos setores econômicos com amplitudes de atuação distintas, o que lhes coloca uma diversidade de atividades e atores econômicos, políticos e sociais. Assim, envolvem a participação e a interação não apenas de empresas e suas variadas formas de representação e associação, como também de diversas outras instituições públicas e privadas voltadas para: formação e capacitação de recursos humanos; pesquisa, desenvolvimento e engenharia; política, promoção e financiamento.

Oaprendizado interativo constituifontefundamental paraa transmissão de conhecimentos e a ampliação da capacitação produtiva e inovativa das firmas e instituições (MYTELCA; FARINELLI, 2000). A capacidade inovativa possibilita a introdução de novos produtos, processos e formatos organizacionais, sendo essencial para garantir a competitividade dos diferentes atores locais, tanto individual como coletivamente.

A governança está relacionada aos diferentes modos de coordenação entre os agentes e as atividades, que envolvem da produção à distribuição de bens e serviços, assim como o processo de geração, disseminação e uso de conhecimentos e de inovações. 0 bom desempenho do cluster depende, em boa parte, da existência de um sistema de governança eficiente e efetivo (SUZIGAN; GARCIA; FURTADO, 2007).

De modo geral os clusters possuem características similares, mas existem diferenças entre eles, seja no perfil, no grau de desenvolvimento ou de estruturação, o que lhes confere características distintivas. Mytelca e Farinelli (2000) apresentam uma tipologia que divide os clusters em informais, organizados e inovativos.

Os clusters informais são mais voltados para o mercado interno, formados principalmente por micro e pequenas empresas, sem uma liderança evidente. Nos clusters informais as relações estabelecem-se sob baixa confiança e cooperação e alta competição, apresentando nível tecnológico baixo, pequena capacidade inovativa e pouco ou nenhum desenvolvimento de novos produtos. Os clusters organizados encontram-se em um estágio intermediário de desenvolvimento, formado por empresas um pouco maiores e com um maior direcionamento para o comércio internacional. A relação entre os agentes se torna mais densa e a capacidade inovativa aumenta. Já os clusters inovativos, por sua vez, são os mais maduros e desenvolvidos, são formados por empresas de todos os portes que apresentam um comportamento mais cooperativo e com alto direcionamento para a exportação, apresentando também uma contínua capacidade inovativa, observa-se no Quadro 1 abaixo. 
Quadro 1: Tipologia para Clusters

\begin{tabular}{|l|l|l|l|}
\hline Variáveis & Clusters informais & Clusters organizados & Clusters inovativos \\
Existência de liderança & Baixa & Baixa e média & Alta \\
Tamanho de empresas & Micro e pequena & PMEs & PMEs e grandes \\
Capacidade inovativa & Pequena & Algumas & Contínua \\
Confiança interna & Pequena & Alta & Alta \\
Níveis de tecnologia & Pequeno & Médio & Médio \\
Linkages & Algum & Algum & Difundido \\
Cooperação & Pequena & Alguma a alta & Alta \\
Competição & Alta & Alta & Média e alta \\
Novos Produtos & Poucos - nenhum & Alguns & Continuamente \\
Exportação & Pouca - nenhuma & Média a alta & Alta \\
\hline
\end{tabular}

Fonte: Mytelca e Farinelli (2000)

Porter (1999) afirma que as vantagens em termos de inovação se instalam no aglomerado em grande parte devido a simples pressão, que pode ser de ordem competitiva por parte dos pares ou fruto da comparação entre os agentes. Isto aliado a similaridade das circunstâncias básicas (como os custos de mão-de-obra e instalações e presença de muitos rivais), reforçam a necessidade das empresas de se distinguir de forma criativa. Portanto, os clusters podem contribuir para a difusão de competências e inovações em um local específico promovendo a economia regional.

A proximidade física das firmas possibilita a redução de custos e a estrutura da rede pode favorecer o fluxo de conhecimento entre organizações em uma região (TAKEDA et al., 2008). Dessa forma, é necessário o estímulo à ação conjunta através de apoio e intervenção em áreas estratégicas, proporcionando o desenvolvimento de inovações (SCHMITZ; NADVI, 1999). Os clusters possuem características próprias direcionadas à inovação, todavia, sua ação conjunta com o Estado e com instituições de ensino e pesquisa podem potencializar ainda mais este perfil. A parceria entre empresas, Estado e Universidades no desenvolvimento de inovações é contemplado pelo Modelo Tríplice Hélice e sua aplicação no estudo de clusters mostra-se muito apropriado.

\section{REDES DE INOVAÇÃO E O MODELO TRÍPLICE HÉLICE}

As formas de parceria interorganizacional têm cada vez mais se tornado um componente central na estratégia corporativa. Segundo Powell (1990), Rosenbloom e Spencer (1996), Roberts e Liu (2001), Chesbrough (2003) e Powell e Grodal (2004), o modelo de redes de inovação encontra-se entre os mais comentados nas duas últimas décadas do século XX. 
No contexto de globalização e rápidas transformações tecnológicas, "a cooperação não é apenas uma maneira de dividir custos e recursos, mas constitui uma apólice de seguro contra alguma decisão errada sobre tecnologia" (CASTELLS, 2007, p.252). Castells (2007) chama a atenção para o fato de que, principalmente nas indústrias de alta tecnologia, alianças, acordos e joint ventures estão mais recorrentes, proporcionando para estas empresas um caminho orientado para a inovação e a vantagem competitiva. Complementando, Beckman e Haunschild (2002) salientam que a colaboração permite que as firmas tenham maior heterogeneidade de portfólio, aprendizado a partir de um estoque mais amplo de conhecimentos, exposição a mais experiências, diferentes competências e oportunidades.

A ideia das redes de inovação se baseia na concepção de que há a necessidade de tipos alternativos de organizações para lidar com diferentes condições de mercado e tecnologia. Sobretudo, nos campos onde os progressos científicos e tecnológicos estão crescendo rapidamente, as fontes de conhecimento se apresentam amplamente distribuídas, de forma que nenhuma firma sozinha detém todas as habilidades para trazer significativas inovações para o mercado (POWELL; GRODAL, 2004). Em virtude disso, os relacionamentos interorganizacionais surgem, cada vez mais, como forma para lidar com a crescente complexidade do conhecimento e da inovação.

Desta forma, as redes, em vez de firmas individuais, têm se tornado o lócus da inovação. As redes como centros de inovação são caracterizadas pela geração e teste de novas idéias em um contexto flexível e colaborativo, construído pela interação entre atores heterogêneos que podem estar dispersos em nível regional, nacional e global. Sua ocorrência, portanto, pode tanto se dar numa região geograficamente concentrada quanto se manifestar de forma descentralizada (DHANARAJ; PARKLE, 2006; PELLEGRIN et al., 2007; QUEIROZ, 2006).

As redes de inovação possuem três implicações chaves para os agentes que as constituem. São elas: a) constituem um dispositivo de coordenação que possibilita e apóia a aprendizagem inter- empresarial; b) permitem a exploração de complementaridades fundamentais para dominar soluções tecnológicas caracterizadas pela complexidade e diversidade de áreas de conhecimento envolvidas; e, c) constituem um ambiente organizacional (ou interorganizacional) que abre a possibilidade da exploração de sinergias pela junção de diferentes competências tecnológicas (BALESTRO, 2004).

As redes, realmente, são um espaço propício para a inovação, porém, representam apenas um dos elementos que favorecem a criatividade, a idealização e a construção de novas ideias. Como destacam, a inovação não é uma peça de um só ato, o que se traduz na necessidade de identificar as estâncias institucionais envolvidas ou que podem se envolver com o processo inovativo (FAGERBERG, 2004; NONAKA; TAKEUCHI, 1997).

As instâncias institucionais a que Fagerberg (2004) se refere são universidades, empresas e governo, constituindo o que ficou entendido como Modelo Tríplice Hélice (Triple Helix Statement). Neste modelo: 
os atores envolvidos (setor científico, setor produtivo e setor público) desempenham papéis vitais na infraestrutura da produção de conhecimento, identificando a dinâmica do processo de inovação, caracterizado pelas diversas etapas necessárias ao desenvolvimento de um produto ou processo inovador. (TERRA, 2001, p.7-8).

Observa-se assim, a mudança de uma interação anteriormente bipolar - universidade e empresa - para uma interação multipolar, envolvendo diversos níveis governamentais.

Neste sentido, os governos "estão oferecendo incentivos, por um lado, e pressionando as instituições acadêmicas, por outro, para que estas desempenhem um papel maior na inovação" (TERRA, 2001, p.4). Logo, o Modelo Tríplice Hélice consiste em transformar o conhecimento de ciência (logo), produzido no ambiente acadêmico, em algo passível de absorção social como techné, evitando que as universidades se tornem "torres de marfim", ou seja, dedicadas à busca do conhecimento por si só (MOWERY; SAMPAT, 2004).

De acordo ainda com Terra (2001), inicialmente baseada no modelo de inovação linear, a contribuição da Universidade para a sociedade era de conhecimento acadêmico. No entanto, no Modelo Tríplice Hélice a inovação é vista como não-linear, podendo ocorrer na relação interinstitucional de organizações de natureza distintas. Tais relações vão desde práticas profissionais esporádicas até vínculos institucionais mais intensos, como é o caso de programas de pesquisa cooperativa. Deve-se salientar que, em ambos os casos, "as esferas científica, produtiva e governamental trabalham de forma independente, assumindo papéis diferenciados a cada instante" (TERRA, 2001, p.8).

Lu (2008) buscou avaliar as implicações do Modelo da Tríplice Hélice na criação de conhecimento e inovação na China. Ele identificou que a formação e operação do sistema de produção de conhecimento naquele país refletem as três dimensões do Modelo. Ou seja, o governo executa o controle normativo, a indústria promove a geração de riqueza e as universidades e instituições públicas de pesquisa desenvolvem inovações. Do outro lado, existe a valorização da dinâmica das interações institucionais e dos processos de transformação na criação da economia do conhecimento. $O$ referido autor concluiu que a adoção e promoção de conhecimento e inovação em torno da interação efetiva entre indústria, governo e universidade foi claramente uma opção estratégica do governo chinês para inserir o país entre as nações inovadoras do mundo.

Dessa maneira, reforçam-se a ideia de se adotar novas concepções acerca da produção de conhecimento e de aprendizagem nas sociedades contemporâneas bem como a necessidade da reformulação dos papéis do Estado e da Universidade na busca pela construção de redes nacionais de inovação. Para Cooke (2005) este é um campo que evoluiu e se consolidou nos últimos anos e que promove uma nova dinâmica na interação entre empresas, instituições de ensino e pesquisa e governo. 
Como destaca Castells (2007), a transformação tecnológica a qual o mundo tem presenciado culminou com o surgimento de uma economia informacional global. Nesse novo contexto, as empresas precisaram se adaptar e o formato de rede emerge como um modelo atual e próprio às necessidades do momento. Castells (2007), no entanto, ainda ressalta que a transformação organizacional ocorreu independente da tecnológica, como resposta a necessidade de lidar com um ambiente operacional em constante mudança. No entanto, uma vez iniciada a transformação organizacional esta foi extremamente intensificada pelas novas tecnologias da informação.

Schilling e Phelps (2007) abordam os clusters como redes interorganizacionais e enfocam a capacidade de transmissão e difusão de informações que esse arranjo possui. Segundo eles, as firmas tendem a interagir mais intensamente ou frequentemente com outras firmas que compartilham de uma proximidade ou similaridade, geográfica ou tecnológica.

As relações entre os agentes que formam o cluster podem ser de natureza forte ou fraca, segundo a teoria dos laços sociais de Granovetter (1973). Conforme este autor, quando os laços são fortes, a rede se fundamenta na confiança e no comprometimento. Este é um vínculo que depende de um tempo mais longo e de dedicação das partes da relação.

Nos laços fortes, observam-se várias superposições, o que se apresenta como limites da rede. Um laço fraco é exatamente o oposto desta situação, ocorrendo em contatos pontuais entre os agentes, que não traduzem proximidade e intimidade, regidas por normas sociais estabelecidas conforme a conveniência do momento, sem apresentar maiores preocupações com o longo prazo. A grande contribuição de Granovetter (1973), no entanto, foi explicar melhor, nas situações onde os laços são fracos, que tipos de benefícios a rede em seu aspecto macro poderia obter. A idéia é de que as relações fracas são aquelas que realmente importam para a expansão das redes. Em outras palavras, o que Gravonetter (1973) salienta é a importância da complementaridade entre os agentes da rede. Esta complementaridade representa um fator de ampliação e vantagem que se manifesta por meio de novas fontes de informação e conhecimento.

A importância dos laços no desempenho dos clusters foi estudada por Eisingerich, Bell e Tracey (2010). Os autores perceberam que o alto desempenho de clusters regionais são sustentados pelas (1) a força da rede, (2) a abertura da rede e (3) pela incerteza do ambiente. Eles conseguiram verificar que o desempenho global de um cluster está relacionado à existência de fortes laços entre seus membros aliado a um elevado grau de abertura, que ajuda a contornar as incertezas do ambiente devido ao acesso a diferentes ideias, tecnologias alternativas, experiências e aprendizados diversos, além de outras oportunidades de negócios.

Para Nakano (2005), uma rede possibilita a uma organização maiores contatos e relações diretas e indiretas com diversas organizações, o que pode facilitar a reciprocidade, a aquisição e difusão de conhecimentos. Assim, uma comunicação eficiente é um fator 
estruturador no estreitamento dos vínculos, como também, instrumento pelo qual os laços de confiança podem se tornar mais densos. Tendo-se por base plataformas de Tecnologias de Informação e Comunicação (TICS), acrescente-se a isso a capacidade destes instrumentos de comunicação proverem um diálogo de agentes do mercado (aspecto micro) e da sociedade civil organizada (aspecto macro), por meio dos relacionamentos interpessoais, tal como sugeriu teoricamente Gravonetter (1973).

Desta forma, o cluster se apresenta favorável ao processo de inovação porque fundamenta suas práticas a partir de uma localidade, isto é, um espaço geograficamente delimitado e estruturado por laços fortes. Neste contexto, a concentração geográfica, as externalidades e informações rápidas propiciam uma geração de valor local própria do espaço em que se circunscreve. A consequência disto é o desenvolvimento de vantagem competitiva para seus membros e para a região onde está instalado (Figura 1).

Figura 1: O local e o global no processo de inovação dos clusters

CLUSTER

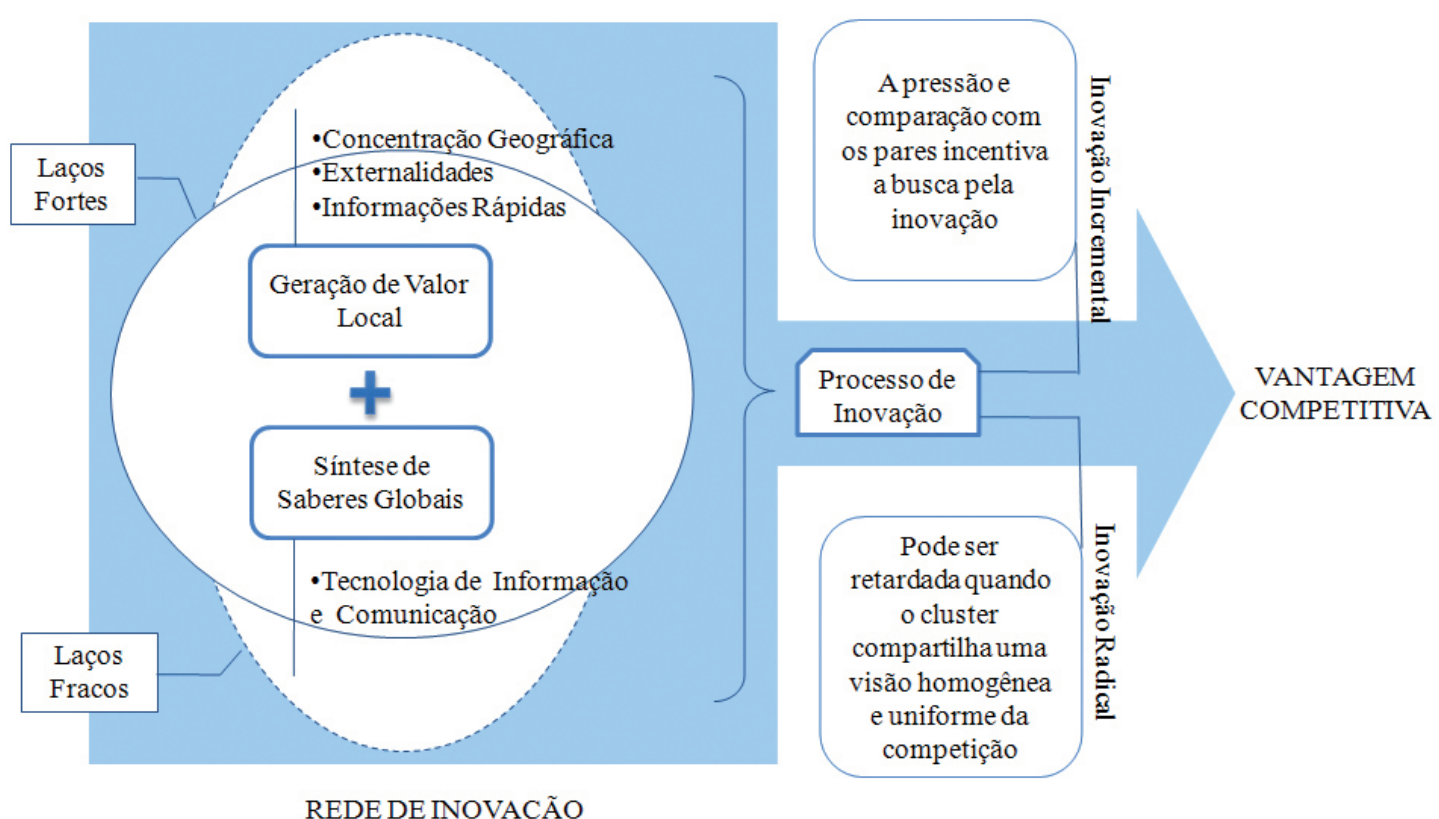

Fonte: Dos autores (2012)

O modelo apresentado engloba múltiplos elementos acerca do processo de inovação, que conjugados podem representar pontos norteadores para futuros estudos. Especialmente no que tange investigações que buscam aprofundar a cerca da interação entre aspectos locais e globais bem como os resultados sobre o processo de inovação incremental ou radical. 


\title{
5 CONSIDERAÇÕES FINAIS
}

Os Clusters são um tipo de arranjo que possui características que contribuem com a vantagem competitiva dos seus membros e do grupo como um todo, além de ser interessante para o desenvolvimento local. Esta capacidade relaciona-se ao potencial de formação de rede de inovação em sua estrutura. A combinação destes agentes, empresas, governo e universidades, em torno de uma atividade produtiva e com interesse no desenvolvimento de inovações, correspondem ao Modelo da Tríplice Hélice.

Este tipo de formação possui o potencial necessário para a geração de valor local, pois apresenta forte identificação regional que lhe faz buscar soluções para problemas específicos, além de fortalecer e utilizar os diferenciais competitivos característicos do grupo para se projetar no mercado. Por outro lado, o cluster age na síntese de saberes globais, uma vez que tem condições melhores do que uma empresa isolada de captar e processar informações.

Desta forma, tem-se que os clusters inovativos permitem a formação de uma rede de inovação que explora as competências locais e o potencial global no desenvolvimento de sua vantagem competitiva. O que distingue um cluster de outro e propicia uma melhor participação de mercado para seus produtos é sua capacidade de explorar sua identidade e características regionais e aproveitar o conhecimento e as informações que estão circulando fora de seu contexto para criar algo novo e se diferenciar dos demais.

\section{INNOVATION NETWORKS IN CLUSTERS: LOCAL AND GLOBAL IN THE CREATION OF COMPETITIVE ADVANTAGE}

\begin{abstract}
Clusters are organizational forms which present intertwined relations between public and private agents around a productive segment in a determined region, which has been considered as source of competitive advantage for firms and regions. This model highlights a favorable environment to innovation development and quality and productivity improvement, especially for giving rise to innovation networks to handle with different market and technology conditions. The innovative process, which is favorable to networks, can be enhanced by the involvement of university-firm, components of Triple Helix Statement. The purpose of this work is to present innovative Clusters as forms capable of offering competitive advantage by means of the relationship between global and local contexts. We consider that the network extent and its characteristics
\end{abstract}


affect Clusters' performance once environments become more uncertain. That is both the network strength and the opening degree of network can be beneficial for Clusters. We argue that firms' capacity to manage the balance between the force and articulation of an open network is a basic source of sustainable and competitive of the Cluster. We observed that public institutions can perform an important role to facilitate the development of new knowledge and consequently innovation. This paper approaches the characteristics of the Clusters that are influential to the competitive advantage of the agents which it constitutes; presents the concepts and characteristics of innovation networks and the role of Triple Helix Statement on its constitution and also deals with the potential of local context to give competitive advantage in a globalization context.

Key-words: Cluster. Triple Helix Statement. Innovation.

\section{REFERÊNCIAS}

ALBAGLI, S.; BRITO, J. Arranjos produtivos locais: uma nova estratégia de ação para o SEBRAE - Glossário de Arranjos e Sistemas Produtivos e Inovativos Locais. [S.I.], Rede de Pesquisas em Sistemas Produtivos e Inovativos Locais (REDESIST), 2003.

AMATO NETO, J. A. Redes de Cooperação Produtiva: uma revisão conceitual. In: AMATO NETO, J. (Org.). Redes de cooperação produtiva e clusters regionais: oportunidades para as pequenas e médias empresas. São Paulo: Atlas, 2000.

BALESTRO, M. V. Redes de Inovação e Capital Social: Apontamentos Conceituais. In: ENCONTRO NACIONAL DOS PROGRAMAS DE PÓS-GRADUAÇÃO EM ADMINISTRAÇÃO, 28, 2004, Curitiba. Anais... Curitiba, 2004.

BECKMAN, C.; HAUNSCHILD, P. Network learning: the effects of partner's heterogeneity of experience on corporate acquisitions. Administrative Science Quarterly, v.47, p. 92124, 2002.

CASTELLS, Manuel. A sociedade em rede. In: CASTELLS, M. A era da informação: economia, sociedade e cultura. São Paulo: Paz e Terra, 2007. p. 209-259.

$\mathrm{CHESBROUGH}, \mathrm{H}$. The era of open innovation. Boston: Harvard Business School Press. 2003.

CHRISTENSEN, H. K. Estratégia corporativa: gerenciando um conjunto de negócios. In: FAHEY, L. (Org.). MBA: curso prático de estratégia. Rio de Janeiro: Campus. 1999. 
COOKE, P. Regionally asymmetric knowledge capabilities and open innovation exploring 'Globalisation 2' - A new model of industry organisation. Research Policy, n.34, v.8, p. 1128-1149, oct. 2005.

DHANARAJ, C.; PARKHE, A. Orchestrating Innovation Networks. Academy of Management Review, v. 31, n. 3, p. 659-662, 2006.

DUTRA, I. F. Projeto de capacitação e consultoria em tecnologia da informação: estudo de caso da aplicação do projeto em arranjos produtivos locais. E-Tech: Tecnologias para Competitividade Industrial, Florianópolis, v. 3, n. 1, p. 63-78, 1ㅇ Sem., 2010.

EISINGERICH, A. B.; BELL, S. J.; TRACEY, P. How can clusters sustain performance: the role of network strength, network openness, and environmental uncertainty. Research Policy, v. 39, p. 239-253, 2010.

FAGERBERG, J. Innovation: a guide to the literature. In: FAGERBERG, J.; MOWERY, D. C., NELSON, R. R. (Orgs.). The Oxford Handbook of Innovation. Oxford: Oxford University Press. 2004.

GRANOVETTER, M. S. The strength of weak ties. American Journal of Sociology, v. 6, p. 1360-1380, 1973.

JENKINS, M.; TALLMAN, S. The shifting geography of competitive advantage: clusters, networks, and firms. Journal of Economic Geography, v. 10, p. 599-618, 2010.

LU, L. Creating knowledge-based innovation in China: the strategic implications of triple helix model. Journal of Technology Management in China, v. 3, n.3, p. 249-263, 2008.

MARSHALL, A. Principles of Economics. Eight Edition. London: Macmillan. 1920.

MOWERY, D.; SAMPAT, B. Universities in national innovation systems. In: FARGERBERG, J., MOWERY, D., NELSON, R. (Eds.). The Oxford Handbook of Innovation. [S.I.]: Oxford University Press, 2004.

MYTELKA, L. K.; FARINELLI, F. Local clusters: innovation systems and sustained competitiveness. Nota Técnica $\mathrm{n}$ 5 do Projeto: Arranjos e sistemas produtivos locais e as novas políticas de desenvolvimento industrial e tecnológico. Rio de Janeiro: IE/UFRJ/ BNDES/FINEP/FUJB, 2000.

NAKANO, D. N. Fluxos de conhecimento em redes interorganizacionais: conceitos e fatores de influência. In: AMATO NETO J. (Org.). Redes entre organizações. 1. ed. São Paulo: Atlas, 2005.

NONAKA, I. ; TAKEUCHI, H. Criação de conhecimento na empresa: como as empresas japonesas geram a dinâmica da inovação. Rio de Janeiro: Campus, 1997. 
PELLEGRIN, I. et al. Redes de Inovação: construção e gestão da cooperação pró-inovação. RAUSP, v. 42, p. 313-312, 2007.

PORTER, M. Aglomerados e competição: novas agendas para empresas, governo e instituições. In: PORTER, M. (Org.). Competição: estratégias competitivas essenciais. Rio de Janeiro: Campus. 1999.

POWELL, W. W. Neither market nor hierarchy: network forms of organization. In: STRAW, B. M.; CUMMINGS, L. L. (Eds.) Research in Organizational Behavior, Greenwich, Conn.: JAO Press, v. 12, p. 295-336, 1990.

POWELL, W. W.; GRODAL, S. Networks of Innovators. In: FAGERBERG, J.; MOWERY, D. C.; NELSON, R. R. (Eds.). The Oxford Handbook of Innovation. Oxford: Oxford University, 2004.

QUEIROZ, A. C. S. Modelos Organizacionais para Inovação. In: Moreira, D. M.; Queiroz, A. C. S. (Org.). Inovação Organizacional e Tecnológica. 1. ed. São Paulo: Thomson. 2006.

ROBERTS, E. B. ; LIU, W. K. Ally oracquire? How technology leaders decide. Sloan Management Review, v. 43, p. 26-34. 2001.

ROSENBLOOM, S.; SPENCER, W.J. Engines of Innovation. Harvard: Harvard Business School Press, 1996.

SCHILLING, M. A.; PHELPS, C. Interfirm collaboration networks: the impact of large-scale network structure on firm innovation. Management Science, v. 53, p. 1113-1126, 2007.

SCHMITZ, H.; NADVI, K. Clustering and Industrialization: Introduction. World Development, v. 27, n. 9, p. 1503 -1514, 1999.

SUZIGAN, W.; GARCIA, R.; FURTADO, J. Estruturas de governança em arranjos ou sistemas locais de produção. Gestão \& Produção, São Carlos, v. 14, n. 2, p. 425-439, mai./ago. 2007.

TAKEDA, Y. et al. Na analysis of geographical agglomeration and modularized industrial network in a regional cluster. A case study at Yamagata prefecture in Japan. Thechnovation, [S.I.], v. 28, p.531-539, 2008.

TERRA, B. A transferência de tecnologia em universidades empreendedoras: um caminho para a inovação tecnológica. Rio de Janeiro: Qualitymark, 2001. 


\section{SOBRE OS AUTORES}
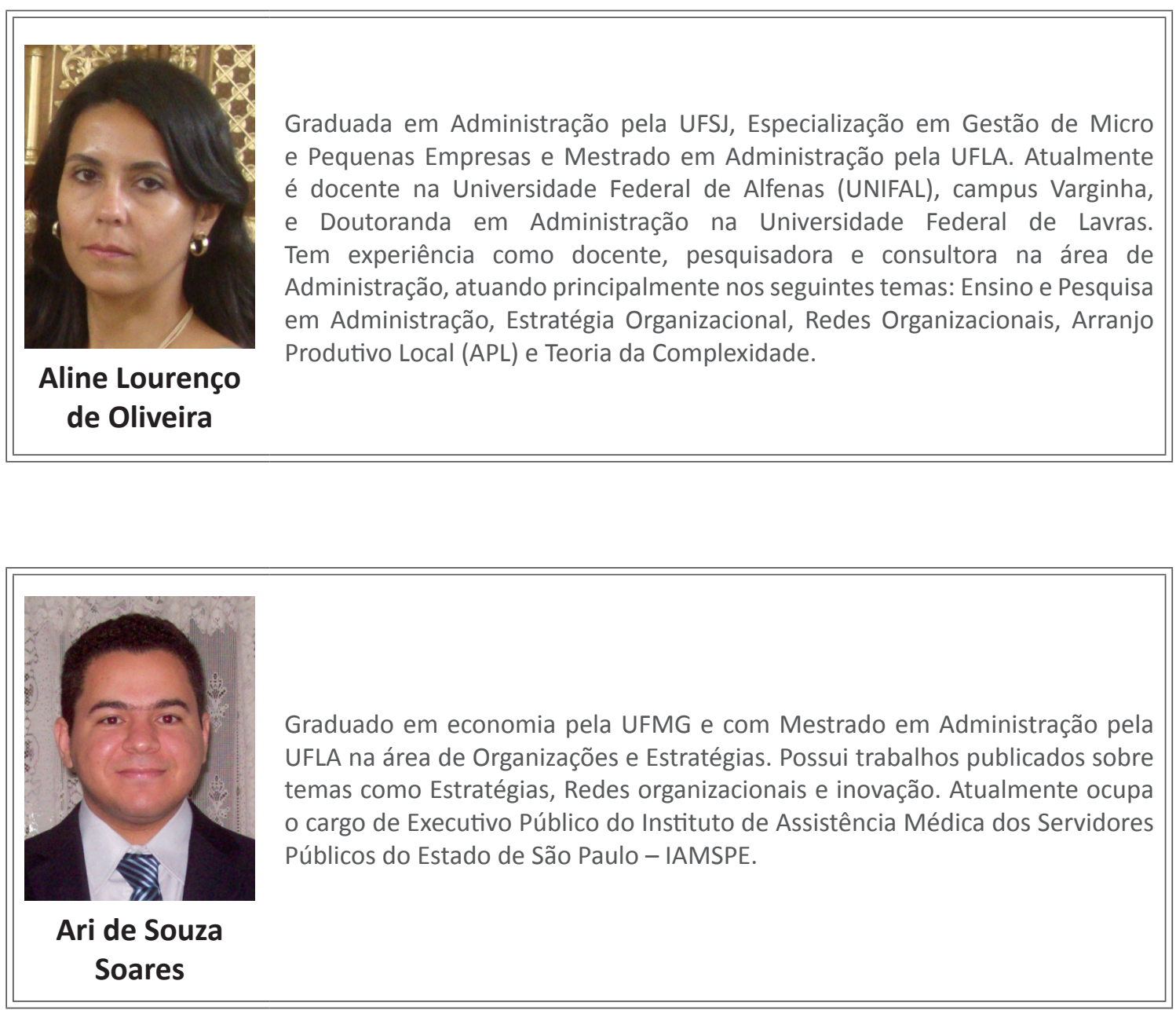

\begin{tabular}{|l} 
Graduado em Administração pela UFLA, Mestrado em Administração e \\
Doutorado em Agronegócios pela UFRGS. Foi coordenador do Programa \\
de Pós-Graduação em Administração da UFLA e Pró-Reitor Adjunto de Pós- \\
Graduação. Atualmente é docente Adjunto da UFLA, Coordenador Institucional \\
da Universidade Aberta do Brasil (UAB), coordenador de projetos do Centro de \\
Educação a Distância (CEAD), líder do Grupo de Estudos em Redes, Estratégia \\
e Inovação (GEREI), orientador de Mestrado e Doutorado, pesquisador da \\
FAPEMIG e CAPES e consultor ad hoc para Avaliação Institucional e de Cursos \\
Superiores do MEC. Tem experiência como docente, pesquisador, gestor \\
educacional e consultor na área de Administração, atuando principalmente \\
nos seguintes temas: Redes de organizações, Cluster, Arranjo Produtivo Local, \\
agronegócio e gestão da tecnologia e inovação.
\end{tabular}




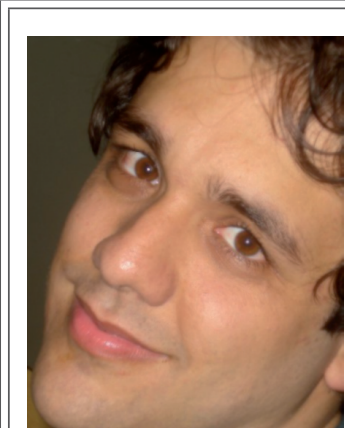

Daniel Leite Mesquita
Graduado em Administração pela Universidade Federal de Lavras (2005), e Mestrado em Administração pela Universidade Federal de Lavras (2009). Atualmente (2010) é Doutorando em Administração pela Universidade Federal de Lavras atuando principalmente nas seguintes áreas: estratégias de organizações, gestão da inovação tecnológica e seus condicionantes. É membro do grupo de estudos em estratégia Redes e inovação - GEREI da Universidade Federal de Lavras cadastrado no diretório do CNPq e tem estudado atualmente os processos de inovações em indústrias no que tange as principais capacidades organizacionais necessárias para o alcance da inovação bem como seus principais mecanismos reguladores. 